\title{
Multiple phakomatoses and primary open-angle glaucoma in one individual
}

\author{
Arijit Mitra, Debarpita Chaudhury, Sumit Choudhury, Suchanda \\ Sar, Smita Ghosh \\ Disha Eye Hospitals, Calcutta, India
}

\begin{abstract}
A 50-year-old woman presented with conjunctival melanosis, scleral pigmentation, and Lisch nodules in her left eye. Intraocular pressure was $24 \mathrm{mmHg}$ in the right eye and 14 $\mathrm{mmHg}$ in the left eye. She had open angles on gonioscopy. Fundus examination showed a cup-to-disc ratio of 0.7 in the right eye, with an inferior notch and a splinter hemorrhage, and 0.6 in the left eye, with a deep cup with sloping rims. Humphrey visual fields showed an evolving superior arcuate scotoma in her right eye; the left eye was normal. Systemic examination showed axillary freckling. The patient had a family history of neurofibromatosis type 1 (NF-1), her father having been diagnosed with the condition. She had hyperpigmentation of the skin over the forehead and periocular skin on the left side. These unique ocular and systemic features were suggestive of two phakomatoses, NF-1 and nevus of Ota, in one eye, and primary open-angle glaucoma (POAG) in the other eye. that is, three pathologies present together in the same individual, which is an extremely rare occurrence.
\end{abstract}

Keywords: conjunctival melanosis, Lisch nodules, nevus of Ota, neurofibromatosis 1, phakomatosis, primary open-angle glaucoma

\section{Introduction}

The presence of primary open-angle glaucoma (POAG) in one eye along with two phakomatoses in the other eye, amounting to three pathologies in the same patient, is an extremely rare phenomenon. In this report, we present a patient with neurofibromatosis type 1 (NF-1) and nevus of Ota in one eye and POAG in the fellow eye.

\section{Case report}

A 50-year-old woman presented to us with the complaint of dimness of vision in her left eye. On examination, her best-corrected visual acuity (BCVA) was 20/20 in the right eye and $20 / 80$ in the left eye. Slit-lamp examination showed conjunctival melanosis and scleral pigmentation in her left eye. She had clear corneas in

Correspondence: Dr. Arijit Mitra, DO, DNB, Glaucoma and Cataract Services, Disha Eye Hospitals, 88 (63 A) Ghoshpara Road, Barrackpore, Calcutta 700120, West Bengal, India. E-mail: jeet2712@yahoo.co.in 


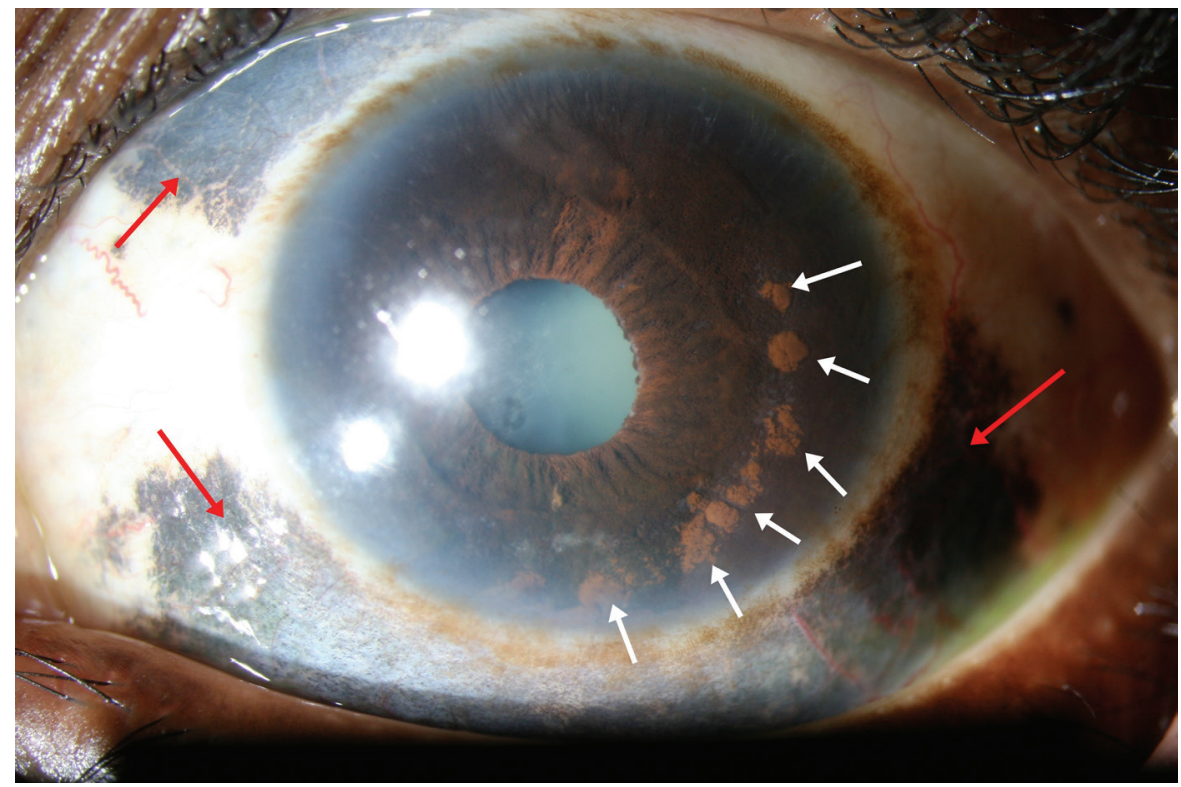

Fig.1. Conjunctival melanosis evident on slit-lamp examination in the left eye of the patient (red arrows). The image also shows iris hyperpigmentation with multiple small, oval or irregular, yellowish-brown, fleshy papules randomly spaced on the inferior and temporal surface of the left iris consistent with Lisch nodules (white arrows).

both eyes. There was normal iris pigmentation in the right eye, but the left eye had hyperpigmented iris with multiple small, oval or irregular, yellowish-brown, fleshy papules randomly spaced on the inferior and temporal surface of the iris consistent with Lisch nodules (Fig. 1).

She had undergone a cataract surgery in her right eye a few years ago a at local institute and there was a posterior chamber intraocular lens (PCIOL) in situ; she had cortical cataract along with posterior subcapsular cataract in her left eye (Fig. 2). Facial examination showed an asymptomatic, hyperpigmented, macular, bluish-black lesion on the skin over her forehead and periocular skin on the left side of her face (Fig. 2e).

Intraocular pressure (IOP) was $24 \mathrm{mmHg}$ in the right eye and $14 \mathrm{mmHg}$ in the left eye; central corneal thickness was $545 \mu \mathrm{n}$ the right eye and $546 \mu \mathrm{n}$ the left eye. She was noted to have Shaffer's grade 4 open angles in both eyes and Scheie's grade I pigmentation of the trabecular meshwork in the right eye. The left eye had diffuse hyperpigmentation of the trabecular meshwork (grade III) and a pigmented Schwalbe's line with patchy pigment deposition in the inferior quadrant. Fundus examination showed a cup-to-disc ratio of 0.7 in the right eye 


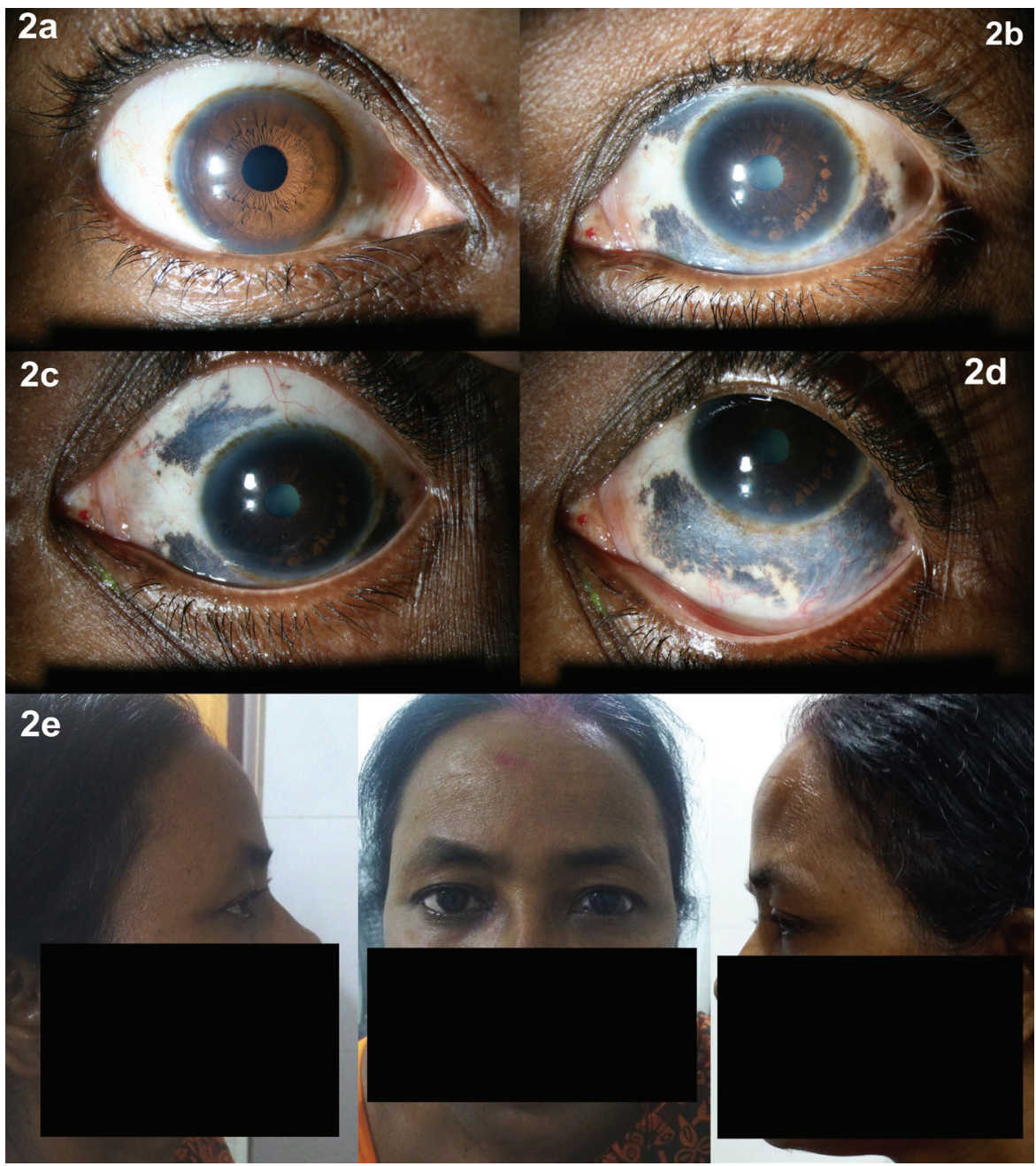

Fig. 2. Anterior segment features of both eyes. (a) The right eye, which had undergone phacoemulsification previously, had a posterior chamber intraocular lens in situ. (b) The left eye in straight gaze clearly shows the Lisch nodules and the pigmentary nevi. The left eye in the downgaze (c) and upgaze (d). (e) Face image of the patient showing evidence of pigmentary nevi in the conjunctiva of the left eye as well as hyperpigmentation of the skin over the forehead and periocular skin on the left side of the face. 
with an inferior notch and a splinter hemorrhage in the inferior aspect of the disc suggestive of glaucomatous damage. The left eye had a cup-to-disc ratio of 0.6 with a deep cup with a sloping, apparently healthy, neuroretinal rim (Fig. 3a). The glaucomatous damage noted in the right eye was recorded to be present before the cataract surgery at another institute where the patient had been under treatment previously. She had been started on a single antiglaucoma medication, travoprost $(0.004 \%)$ once daily, and subsequently underwent cataract surgery in that eye. However, the patient discontinued the antiglaucoma medication herself and was not using any medication for the past 6 months before she presented to us. Ultra-widefield (UWF) imaging was done using using the Optos ${ }^{\circledast}$ (Optos, Dunfermline, UK). Optomap showed normal peripheries in both eyes (Fig. 3b).

Anterior segment optical coherence tomography (AS-OCT) was done using the RTVue (Optovue, Inc., Fremont, CA) and the scans of both eyes were normal. Visual fields done using the Humphrey Field Analyzer (Carl Zeiss Meditec, Dublin, (A, USA) showed an evolving superior arcuate scotoma with a nasal step in her right eye while the left eye was normal (Fig. 3c). OCT (RNFL) using the RTVue showed retinal nerve fiber layer (RNFL) thinning in the inferotemporal and inferonasal quadrants in the right eye, while the left eye had normal RNFL thickness in all quadrants. Magnetic resonance imaging scans of the brain and orbits were normal (Fig. 4).

On physical examination, the patient had axillary freckling but did not have any skin growth, scoliosis, or other skeletal abnormalities. There were no neurofibromas or café-au-lait macules. Her head circumference was within normal range for an adult female $(58 \mathrm{~cm})$, and neither hypertelorism nor ear abnormalities were present. She gave no history of learning disabilities, seizures, or growth and developmental delays. There was a family history of neurofibromatosis type 1 (NF-1), with her father having been diagnosed with the condition. There was no family history of pigmentary abnormalities, or other genetic diseases. Based on the presence of the three features, Lisch nodules, axillary freckling and one parent being a diagnosed case of NF-1, she was diagnosed as a case of NF-1. Since she had the typical features of ocular melanosis in her left eye and dermal melanosis on the left side of her face, she was also diagnosed as a case of Nevus of Ota. The patient's IOP was raised in the right eye and there were glaucomatous changes in that eye on fundus examination confirmed by visual field reports, so she was diagnosed as a case of primary open-angle glaucoma (POAG). Thus, her final diagnosis was NF-1 and nevus of Ota in the left eye and POAG in the right eye; that is, the presence of three coexisting pathologies in the same individual. To the best of our knowledge this is the first case report presenting these three pathologies in one individual.

A prostaglandin analogue (travoprost $0.004 \%$ ) once daily was started in her 


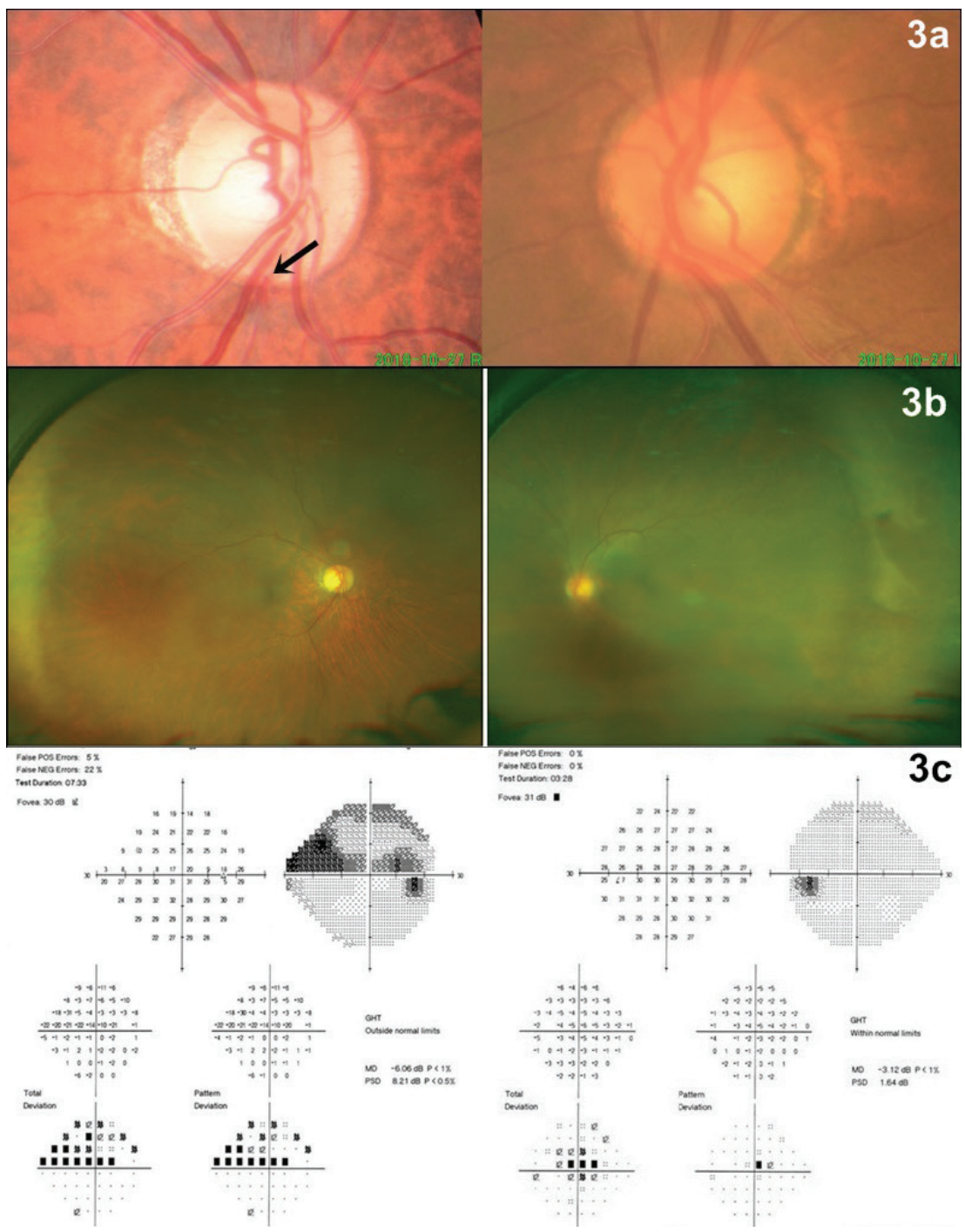

Fig. 3. (a) Disc images. The right eye had a cup-to-disc ratio of 0.7 with an inferior notch and a faint splinter hemorrhage in the inferior aspect of the disc (black arrow). The left eye had a cup-to-disc ratio of 0.6.(b) Wide field fundus image of the patient. The periphery was normal in both eyes. (c) Humphrey visual fields of both the eyes. It reveals an evolving superior arcuate scotoma in the right eye with depressed points in the superior hemifield while the left eye shows a normal field. (d) Optical coherence tomography. The report showed retinal nerve fiber layer (RNFL) thinning in the inferotemporal and inferonasal quadrants in the right eye while the left eye had normal RNFL thickness in all quadrants. 


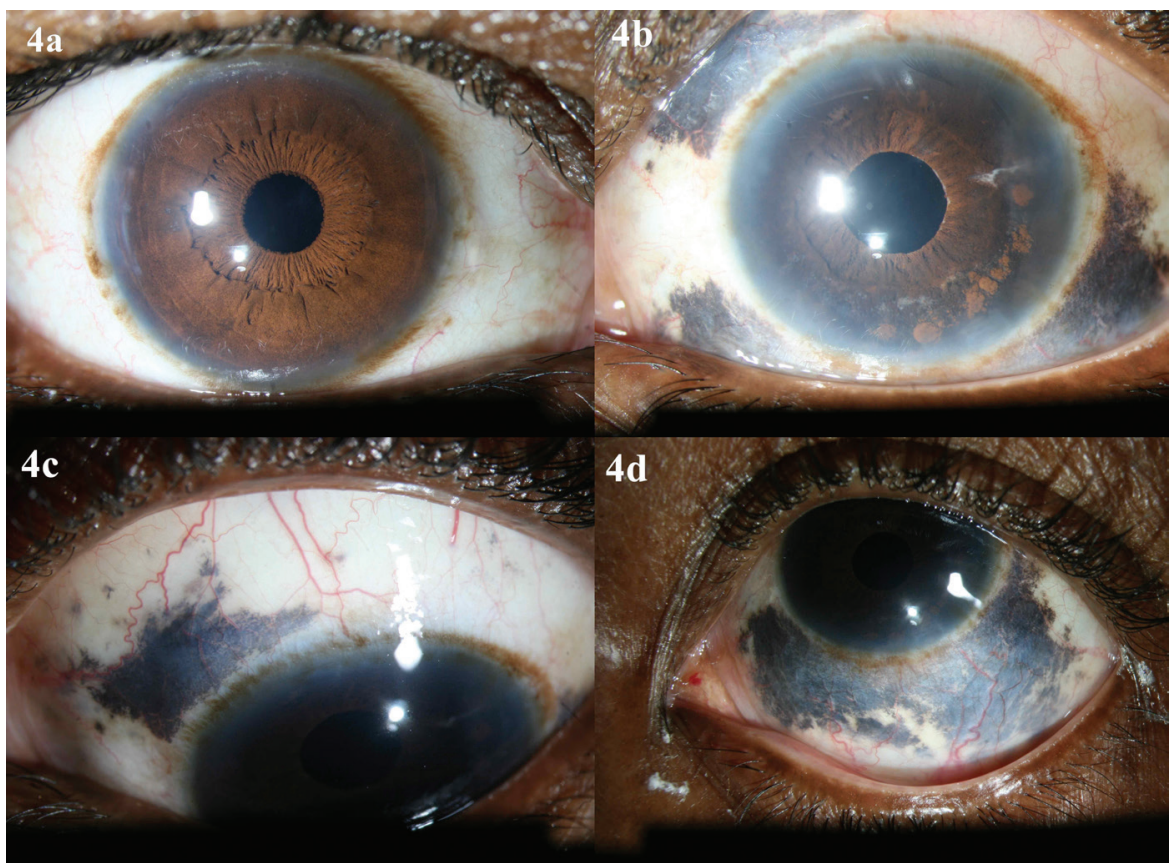

Fig. 5. Anterior segment photographs of the patient at follow-up after cataract extraction in the left eye. Both eyes had intraocular lenses in situ. (a) Right eye. (b, c, d) Left eye in straight, downgaze, and upgaze, respectively.

right eye only. She underwent an uneventful cataract extraction in her left eye, and a multipiece intraocular lens ( $\mathrm{IOL}$ ) was implanted (Fig. 5). During follow-up, IOP was $17 \mathrm{mmHg}$ in the right eye and $15 \mathrm{mmHg}$ in the left eye. Fundus examination revealed the resolution of the splinter hemorrhage in the right eye. She was advised continuation of travoprost in the right eye and regular follow-ups with periodical visual field examinations.

This patient is unique since she had ocular, facial, and dermatological findings suggestive of two phakomatoses in the same individual: nevus of Ota and NF-1, along with the presence of POAG in the other eye. Our literature search showed no published case reports showing the presence of these three pathologies together.

\section{Discussion}

Nevus of Ota or nevus fuscoceruleus ophthalmomaxillaris was first described by Hulke in 1869 before Ota's definitive description in 1939. ' It is a dermal melanocytic hamartoma that presents as bluish-grey or blackish-brown hyperpigmentation 


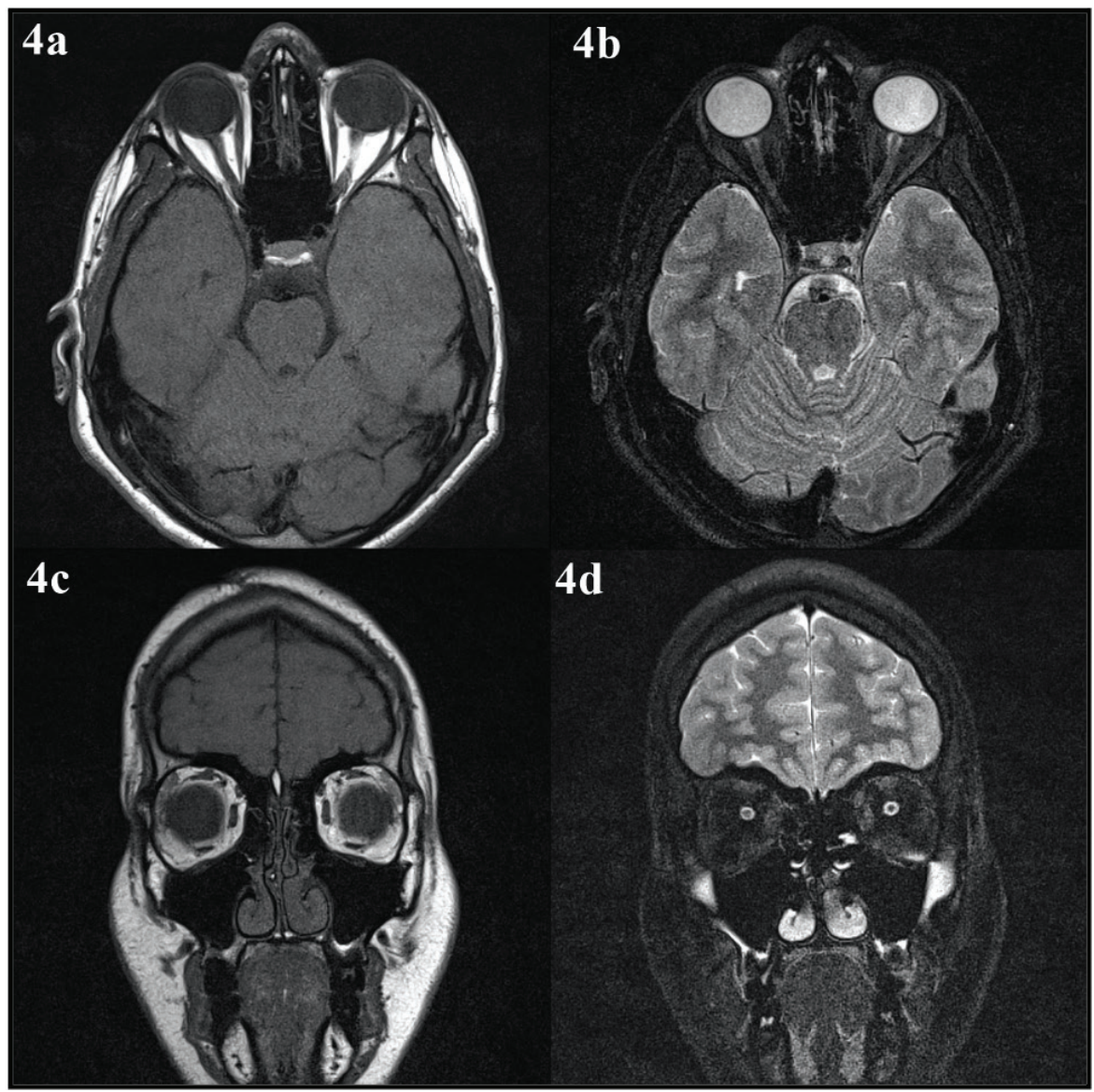

Fig. 4. Magnetic resonance scans of the patient. (a) Axial T1 and (b) axial T2 fat saturation scans showing normal optic nerves, no abnormalities of the bony orbit, and normal brain tissue. (c) Coronal T 1 scan showing normal contour of the bony orbit and normal extraocular muscles. (d) Coronal T2 fat saturation scans which shows normal optic nerves and brain tissue.

on the facial skin limited to the area innervated by the first and second divisions of the trigeminal nerve. An increased number of dermal melanocytes located in the distribution of the ophthalmic and maxillary divisions of the trigeminal nerve has been reported. Most patients have concomitant hyperpigmentation of the globe that may involve the conjunctiva, sclera, cornea, iris, and fundus. In our patient, the involvement of the conjunctiva and sclera were evident. Extracutaneous involvement, especially ocular involvement, is frequent and has been widely reported..$^{2-4}$ 
In nevus of Ota, the melanosis may also affect the oral and nasal mucosa, external auditory canal, tympanic membrane, orbital tissues and brain. In our patient, however, these features were not present. Nevus of Ota affects between $0.014 \%$ and $0.034 \%$ of the Asian population. ${ }^{2}$ The reported prevalence of ocular hyperpigmentation in patients with dermal involvement has ranged from $22 \%$ to $61.3 \% .^{3,4}$ In patients with extensive dermal lesions, ocular involvement is as high as $76.6 \% .^{3,4}$

The pathogenesis of this condition is unknown, but some of the theories which have been put forward include:

1. dropping-off of epidermal melanocytes;

2. migration of hair bulb melanocytes to the dermis; and

3. reactivation of pre-existing latent dermal melanocytes, which are triggered by dermal inflammation, UV radiation, or hormonal changes during pregnancy.3.4

Histopathologically, in nevus of Ota, pigment-bearing cells are numerous and distributed diffusely throughout the upper and lower dermis. These are elongated, slender pigment-bearing cells dispersed in between the collagen fibers of the dermis and the long axis of pigment-bearing cells, that is, melanocytes are found along the collagen fibers. ${ }^{3,4}$

Even though the iridocorneal angle may be extremely hyperpigmented, there may not be any rise of IOP. This has been reported in a few studies and was seen also in our patient. ${ }^{4,5}$ Elevated IOP with or without glaucomatous damage has been reported in $10 \%$ of patients of nevus of Ota with ocular involvement. ${ }^{5}$ The affected eye typically has very heavy pigmentation of the trabecular meshwork and histopathological studies have shown melanocytes in the meshwork. ${ }^{6}$

Lisch nodules are the most common ophthalmologic manifestation of NF-1.,8 On histological examination, they are found to be melanocytic hamartomas, composed of melanocytes, elongated fibroblasts, and mast cells, presumably of neural crest origin, similar to other cutaneous characteristics of NF-1. ${ }^{9}$ Lisch nodules do not represent a cause of morbidity or disability, but they are diagnostically significant, being one of the hallmark manifestations of NF-1. Examination of a Lisch nodule with the help of a slit lamp reveals characteristic nodules with dimensions of approximately $2 \mathrm{~mm}$, absence of vasculature, and a certain chromatic variability ranging from white to yellow or brown. ${ }^{9}$ The differential diagnosis of Lisch nodules includes iris mammillations, iridocornealendothelial syndrome, granulomatous iritis, iris cysts, multiple iris nevi, iris melanoma, and other malformations. ${ }^{10,11}$

Unilateral Lisch nodules are rare but they have been reported in cases of segmental neurofibromatosis, found associated with other pigmentary changes or neurofibromas. ${ }^{12}$ The presence of Lisch nodules without other clinical 
evidence of NF-1 has been reported, although this is also extremely rare. . $^{812,13}$ There are only two reported cases of numerous unilateral Lisch nodules in the absence of any additional features of NF-1..13,14 In our case, the Lisch nodules were also unilateral, but our patient had axillary freckling and a positive family history of NF-1.

Iris mammillations are a very commonly noted abnormality of the iris. They are characterized by deep brown, regularly spaced, smooth, conical iris elevations. ${ }^{10}$ These can be differentiated from Lisch nodules by slit-lamp examination as Lisch nodules are yellowish-brown, dome-shaped, well-defined elevations rising from the surface of the iris. ${ }^{9}$ Amelanotic iris nevi present as flat, or minimally elevated, lightly pigmented lesions with blurred margins which are visibly different from the elevated dome-shaped Lisch nodules. The amelanotic naevi are much lighter in color compared to the yellow or yellowish-brown Lisch nodules. ${ }^{9}$

In NF-1, the various clinical features affecting the nervous, dermatologic, ocular, and skeletal systems are usually absent at birth. Approximately $95 \%$ of NF-1 patients meet the diagnostic criteria by the age of 8 years, and almost all do so by the age of 20 years. The diagnostic criteria currently used for diagnosis were presented by the National Institutes of Health statement in $1987 . .^{15} \mathrm{~A}$ clinical diagnosis is the most common way doctors diagnose NF1. A diagnosis of NF-1 is confirmed if the patient has two or more of the following:

1. six or more café-au-lait spots, at least $0.5 \mathrm{~cm}$ in children or $1.5 \mathrm{~cm}$ in adults;

2. two or more neurofibromas on or under the skin, or one plexiform (deep tissue) neurofibroma;

3. axillary (armpit) or inguinal (groin) freckling;

4. optic pathway glioma, also called a visual pathway tumor;

5. two or more Lisch nodules;

6. bone changes such as bowing of the long bones; and

7. a close relative (parent, child or sibling) with a confirmed diagnosis of $\mathrm{NF}-1 .{ }^{15}$

Our patient had axillary freckling, Lisch nodules, and one parent having been diagnosed as a case of NF-1 and was thus confirmed as a case of NF-1.

The axillary and/or inguinal freckling are commonly seen in patients of NF-1. They usually start as small freckles less than $5 \mathrm{~mm}$ in size and are localized usually in the axillary or inguinal area (Crowes sign). Freckling is found in approximately $90 \%$ of adults over the age of 30 years, ${ }^{15}$ as was seen in our case.

Raised IOP and/or disc changes suggestive of glaucoma may be present at birth in a case of NF-1 and usually suggest congenital abnormality of the angle, while onset at a later date suggests involvement of the anterior chamber angle secondary to NF-1. ${ }^{16-18}$ This may be due to angle infiltration by neurofibroma or 
the ciliary body may show thickening leading to secondary angle closure. ${ }^{16-18}$ Glaucoma has been reported in 1/300 NF-1 patients. ${ }^{19}$ In our patient, there were no signs of glaucoma in the eye affected by the phakomatoses.

The presence of nevus of Ota and NF-1 in one individual is an extremely rare presentation. Our literature search showed that there are only three published case reports of the association of nevus of Ota with NF-1..$^{20-22}$ However there are no published reports of the presence of three pathologies in one individual as was seen in our case.

Medical management is the initial mode of treatment in glaucoma associated with these phakomatoses, as is the case in other forms of open-angle glaucoma. ${ }^{16,17}$ Laser trabeculoplasty may also be attempted in some of these cases. If medical management and laser treatment fail, then trabeculectomy may be tried as a surgical option. ${ }^{17,18}$

POAG is usually bilateral disease but there are a number of published reports of cases of POAG where only one eye has shown glaucomatous damage..$^{23-25}$ Some authors use the term unilateral POAG for these patients. ${ }^{23-25}$ In most of these cases, the other eye will not be affected at all and there are no disc or field changes suggestive of glaucoma in the unaffected eye over long-term follow-up. ${ }^{3-25}$ Our case appears to be a case of unilateral POAG with evident glaucomatous changes in the right eye, with the left eye having phakomatoses. The management of these unilateral POAG patients follows the same management guidelines of POAG cases; the only difference is that the treatment is given to the affected eye only.

This case is different from published reports in two aspects. Firstly, it shows the coexistence of two phakomatoses in one individual, which is an extremely rare finding. However, even though the patient's left eye had the clinical features of two phakomatoses and a hyperpigmented trabecular meshwork, IOP was normal and there were no disc or field changes suggestive of glaucoma. Secondly, this patient had raised IOP and glaucomatous damage in the right eye suggestive of POAG, even though the left eye had the clinical features of two phakomatoses. There are no published reports of the coexistence of these three pathologies in one individual and hence, it is an extremely rare presentation.

\section{Conclusion}

Coexistence of multiple phakomatoses in an individual is rare. There are no specific treatment options for these patients. There may be underlying systemic and/or local anomalies and complications associated with these disorders or other non-associated ocular or systemic diseases which must be diagnosed, which will dictate the management of these patients. Further advancements and research into the molecular biology of neural crest development and 
abnormal migration of melanocytes are warranted to determine the etiology of these coexisting neuro-cutaneous disorders. There may be additional pathologies, such as POAG, along with the existence of phakomatoses in the same individual; thus, thorough examination of these patients and proper management and follow-up are required.

\section{Acknowledgements}

The authors have written consent from the patient to publish the images in this case report.

\section{References}

1. Ota M. Naevus Fusco-caerunleus ophthalmomaxillaries. Tokyo Med J. 1939;63:1243-1245.

2. Stanford DG, Georgouras KE. Dermal melanocytosis: a clinical spectrum. Aust J Dermatol. 1996;37:19-25.

3. Ito M. Studies on melanin. XXII: Naevus Fusco-caeruleus acromiodeltoideus. Tohoku J Exp Med.1954;60:10-18.

4. Hidano A, Kajima H, Ikeda S, Mizutani, Miyasato H, Niimura M. Natural history of Naevus of Ota. Arch Dermatol.1967;95:187-195.

5. Teekhasaenee C, Ritch R, Rutnin U. Leelawongs N. Ocular findings in oculodermal melanocytosis. Arch Ophthalmol. 1990;108:1114-1120.

6. Sugar HS. Glaucoma with trabecular melanocytosis. Ann Ophthalmol.1982;14:374-375.

7. Kinori M, Hodgson N, Zeid JL. Ophthalmic manifestations in neurofibromatosis type 1. Surv Ophthalmol. 2018;63(4):518-533.

8. Huson S, Jones D, Beck L. Ophthalmic manifestations of neurofibromatosis. Br J Ophthalmol.1987;71:235-238.

9. Nichols JC, Amato JE, Chung SM. Characteristics of Lisch nodules in patients with neurofibromatosis type1. J Pediatr Ophthalmol Strabismus. 2003;40:293-296

10. Kharrat W, Dureau P, Edelson C, Caputo G. Iris mammillations: three case reports. J. Fr. Ophtalmol. 2006;29:413-417.

11. Ragge NK, Falk RE, Cohen WE, Murphree AL. Images of Lisch nodules across the spectrum. Eye (Lond). 1993;7:95-101.

12. Ceuterick SD, Van Den Ende JJ, Smets RM. Clinical and genetic significance of unilateral Lisch nodules. Bull. Soc. Belge Ophtalmol. 2005;295:49-53.

13. Lal G, Leavitt JA, Lindor NM, Mahr MA. Unilateral Lisch nodules in the absence of other features of neurofibromatosis 1. Am J Ophthalmol. 2003;135:567-568.

14. Adams EG, Stewart KMA, Borges OA, Darling T. Multiple, Unilateral Lisch Nodules in the Absence of Other Manifestations of Neurofibromatosis Type 1. Case Rep Ophthalmol Med. 2011;2011:854784. doi: 10.1155/2011/854784.

15. Neurofibromatosis. Conference statement. National Institutes of Health Consensus Development Conference. Arch Neurol. 1988;45:575-578.

16. Abdolrahimzadeh S, Plateroti AM, Recupero SM, Lambiase A. An Update on the Ophthalmologic Features in the Phakomatoses. J Ophthalmol. 2016:3043026. Epub: 2016 Jul 17.

17. Jain G, Jain VK, Sharma IK, Sharma R, Saraswat N. Neurofibromatosis Type 1 Presenting with Ophthalmic Features: A Case Series. J Clin Diagn Res. 2016;10(11):SR01-SR03. Epub: 2016 Nov 1.

18. Grant WM, Walton DS. Distinctive gonioscopic findings in glaucoma due to neurofibromatosis. Arch Ophthalmol. 1968;79:127-134. 
19. Morales J, Imtiaz AC, Bosley TM. Glaucoma and globe enlargement associated with neurofibromatosis type1. Ophthalmology. 2009;116:1725-1730.

20. Croxatto JO, Charles DE, Malbran ES. Neurofibromatosis associated with nevus of Ota and choroidal melanoma. Am J Ophthalmol. 1981;92:578-80.

21. Gupta A, Ram J, Jain IS. Nevus of Ota associated with neurofibromatosis. Ann Ophthalmol. 1986;18:154-155.

22. Madke B, Kar S, Gangane N, Singh N. Phacomatosis Cesioflammea in Association With von Recklinghausen Disease (Neurofibromatosis Type I). Cutis. 2017;99(2):E35-E37.

23. Sangawe JL. Unilateral glaucoma. Trop Geogr Med. 1986;38(1):70-72.

24. Yoo YJ, Lee EJ, Kim T-W. Intereye difference in the microstructure of parapapillary atrophy in unilateral primary open-angle glaucoma. Invest Ophthalmol Vis Sci. 2016;57:4187-4193.

25. Zangalli CS, Ahmed OM, Waisbourd M, et al. Segmental Analysis of Macular Layers in Patients with Unilateral Primary Open-Angle Glaucoma. J Glaucoma. 2016;25:401-407. 